\title{
Interactive comment on "The role of dew as a nighttime reservoir and morning source for atmospheric ammonia" by G. R. Wentworth et al.
}

\section{Anonymous Referee \#2}

\section{Received and published: 9 May 2016}

This manuscript examines the potential for ammonia accumulation in dew with subsequent release to the atmosphere during surface drying. The authors examine this process using a combination of laboratory and field dew chemistry measurements, measurement of dew amount, and atmospheric measurements of ammonia. The experiment is well designed and the results and conclusions are, for the most part, well supported by the data, which are of high quality. The manuscript is well written and appropriate for Atmospheric Chemistry and Physics. I recommend publication subject to treatment of the following comments.

Page 3, Line 30: "Most larger scale (regional or global) chemical transport models (CTMs) still employ..." The authors should acknowledge that the models are evolving. For example, CTMs commonly used for North America contain a bidirectional framework for NH3 fluxes (see Pleim et al, 2013, doi:10.1002/jgrd.50262; Zhang et al., 2010 
doi: 10.1029/2009JD013589).

Rain measurements should be briefly described in Materials and Methods.

Page 13, Line 11: “...NH3 deposited into dew overnight should not necessarily be counted towards the total $\mathrm{N}$-deposition budget. ... This is an important statement (and there is a similar statement on Page 14, Lines 25-27) that begs the question, how might the dew measurements differ from real processes in both the grass field and surrounding forest? Is it reasonable to expect that some dew is transferred from the canopy to the ground where the $\mathrm{NH} 4+$ would be more likely to remain in the ecosystem? How might the amount and timing of dew in the grass field differ from the surrounding forest?

Regarding the interpretation of the atmospheric measurements of $\mathrm{NH} 3$, it is likely that during some periods the emission footprint driving the variability in atmospheric $\mathrm{NH} 3$ extends well outside of the grass field in which the dew measurements were made. This may be further complicated by topographically induced advection of $\mathrm{NH} 3$ from upslope/downslope as well. For these reasons, and because the field is surrounded by forest, some discussion of the representativeness of the measurements relative to the larger surrounding ecosystem is warranted.

Discussion of $\mathrm{NH} 3$ loss rates beginning Page 16, Line 9. The finding of similar loss rates on dew and dry nights is interesting and to me a bit surprising. The implication that dew results in a net lower deposition flux to the ecosystem is important from both a budget standpoint and process modeling. The calculated loss rates, assumed to reflect deposition, are based on a mass balance framework that may be considerably more complicated in complex terrain. For example, the rate could be affected by advection of $\mathrm{NH} 3$ depleted air from upslope rather than deposition. In my opinion, this aspect of the paper would benefit from further analysis. The authors should consider including some discussion of meteorological conditions associated with dew versus dry nights. Are wind speed and direction similar? Regarding NH3 deposition processes, I agree with the suggestion that $\mathrm{Ra}$ and $\mathrm{Rb}$ dominate over the surface resistance at 
night. This is another instance where an examination of meteorology may be helpful. Comparison of $\mathrm{Ra}$ and $\mathrm{Rb}$ on dew versus dry nights would provide some insight into potential differences in exchange processes. Were the CASTNET meteorological measurements active during the study period or were there other measurements from which Ra and Rb may be calculated? If not, even a basic analysis of wind speed and direction during dew versus dry nights would be informative. The results suggest that, assuming the atmospheric resistances are similar on dew versus dry nights, similar rates of non-stomatal deposition occur when the surface is wet versus dry. Can the authors speculate regarding the "dry" process? Of the nights with no surface wetness presented in figure $4 \mathrm{~b}$, what were typical maximum values of relative humidity?

Interactive comment on Atmos. Chem. Phys. Discuss., doi:10.5194/acp-2016-169, 2016. 